\section{Flujo salival y caries corono-radicular en pacientes adultos mayores}

\author{
Salivary flow and corono-radicular caries in elderly patients
}

\section{Resumen}

El objetivo del estudio, fue determinar la correlación entre el flujo salival y caries corono-radicular de personas adultos mayores, donde se analizó las propiedades físico-químicas de la saliva (flujo, $\mathrm{pH}$ ), la higiene, la frecuencia y ubicación de las caries dental corono-radicular. Se seleccionó una muestra de 45 personas mayores de 60 años y otra de 18-26 años (grupo control), provenientes de los centros de asistencia, hospitales y la universidad, esto nos permitió determinar la diferencia del flujo salival en los dos grupos etareos. La técnica de recolección de saliva, consistió en hacer que mastiquen una pastilla de parafina por un tiempo de cinco minutos y que paulatinamente escupieron en un depósito; que permitió medir el volumen y el $\mathrm{pH}$ salival. Tambien se determinó la higiene bucal y observamos la caries corono - radicular de todas las caras de los dientes. El volumen salival promedio en adultos mayores fue $3,27( \pm 2,4)$ mientras que en los jóvenes fue $5.24( \pm 2,4)$, el flujo salival promedio en los adultos mayores fue de $0,65 \mathrm{ml} / \mathrm{min}( \pm 0,48)$ muy inferior al de las personas jóvenes en los que se halló un promedio de $1.05 \mathrm{ml} / \mathrm{min}( \pm 0,47)$, el $\mathrm{pH}$ salival. con una media de $7,10 \mathrm{y} \pm 0,37$. Se encontró que $98 \%$ las personas de tercera edad mostraron mala higiene bucal. El promedio de dientes presentes en boca de los adultos mayores fue de 16 piezas $\pm 6,59$, un $95 \%$ estaban afectadas por caries con una media de $5,8( \pm 3,34)$.

Debido a la deficiente higiene bucal no se pudo determinar la correlación entre el flujo salival y la caries corono-radicular; motivo de estudio, no se pudo determinar.

Palabras Clave: Flujo salival, $\mathrm{pH}$ salival, caries corono-radicular, adultos mayores.

\section{Abstract}

The aim of this study was to determine the correlation between hypo-salivation and corono-radicular caries in older adult patients, which analyzed the physicochemical properties of saliva (flow, $\mathrm{pH}$ ), the frequency and location of the corono-radicular caries. Was selected a sample of 45 persons, adult patients over 60 years and another 18 to 26 years (control group), from service centers, hospitals and universities. The technique of collection of saliva, consisted of making chewing a piece of paraffin for a period of five minutes and gradually spit in a tank, which allowed to measure the volume and $\mathrm{pH}$ salival. We observed the oral higiene and the corono-radicular caries of all tooth surfaces. The results, the volume of saliva in the elderly, had an average of $3.27 \mathrm{ml} / 5 \mathrm{~min}$ very low values compared with the values of young people who have an average of $5.22 \mathrm{ml} / 5 \mathrm{~min}$. It was found that $98 \%$ of seniors showed poor oral hygienes. On crown-root caries, the average mouth of teeth present in older adults was 16 pieces, but these pieces by $95 \%$ were affected by decay with a mean of 5.8 and \pm 3.8 . On the relationship between hyposalivation and dental caries, could not be determined, since the plaque was very high at $98 \%$, it was observed that older adults are at high risk of corono-radicular caries. To avoid this severe pathology is necessary to intervene in that age group.

Key words: salivary flow, salivary $\mathrm{pH}$, corono-radicular caries, elderly patients.

\section{Artículo ORIGINAL}

\section{Carlos Campodónico \\ Reátegui', Sylvia Chein \\ Villacampa ${ }^{1}$, Lourdes \\ Benavente Lipa ${ }^{1}$, Juana \\ Delgadillo Ávila², María \\ Angélica Alvarez Paucar ${ }^{3}$, \\ Sofía Espinoza Escajadillo², \\ Raúl Vidal Goñí1, María \\ Ventocilla Huasupoma ${ }^{1}$, Tereza Evaristo Chiyong', Carla Campodónico Morales ${ }^{4}$}

\footnotetext{
1 Departamento Académico de Estomatología preventiva y Social

2 Ciencias básicas

3 Odontopediatria

4 Egresada de Segunda Especialidad de Ortodoncia y Ortopedia
}

Correspondencia:

Mg. Carlos Campodónico Reátegui

Facultad de Odontología. Av. Germán Amezaga s/n, Lima, 1 Perú.

Teléfono: 6197000 an 3404

Correo electrónico: ccampo_04umber@hotmail. com

Fecha de recepción: 03-08-12

Fecha de aceptación: 15-11-12

\section{Introducción}

La saliva es un elemento influyente en el proceso carioso, es la razón por la cual constituye el estudio de este fluido un factor importante en la búsqueda de elementos concretos que permitan relacionar las propiedades y composición físicas y química de la saliva con enfermedades que afecten la cavidad bucal ${ }^{1,2,3,4}$ También la saliva puede ser empleada como medio de diagnóstico odontológico o médico. ${ }^{5,15}$

Barrancos, refiere que la saliva cumple con varias funciones como: protectora, lubricación, antimicrobiana (proteínas salivales: lisozima, lactoferrina, lactoperoxidasa, mucina cistatina, histatina, IgA secretoria, glucoproteína rica en prolina), integridad de la mucosa, buffers (bicarbonato, iones fosfato, péptidos ricos en histidina) Remineralización dentaria (calcio, fosfato, estaterina, proteínas ricas en prolina aniónica. ${ }^{6}$

Para Sreebny la xerostomía se asocia frecuentemente a una disminución del flujo salival (hiposalivación). Sin embargo, no todos los casos de hiposalivación se acompañan de xerostomía o boca seca. Se considera que existe un flujo salival mínimo, por debajo del cual el paciente casi siempre se queja de boca seca. Este flujo, en reposo, es de $0,1-0,2 \mathrm{ml} /$ min de saliva total; en estado de estimulación, el valor asciende a 0,5-0,7 $\mathrm{ml} / \mathrm{min}^{7}$. Para una hiposalivación con un flujo salival $\leq 0,2 \mathrm{ml} / \mathrm{min}$ sería necesario que estuviera afectado alrededor del $50 \%$ del parénquima glandular. ${ }^{8,15}$ Krasse B. considera que cuando el rango de secreción salival está reducido, las lesiones cariosas a menudo aparecen en la unión cemento-esmalte. En general, tales lesiones cariosas pueden ser distinguidas fácilmente por la erosión o 
reabsorción idiopática, estas lesiones, como norma tienen una base dura. Según McNamara la incidencia de caries radicular aumenta a medida que la población envejece y el edentulismo es menos prevalente debido al aumento en la conciencia del cuidado dental, ya que las exposiciones radiculares por recesión gingival o pérdida del nivel de inserción, han aumentado con el envejecimiento. ${ }^{10}$ Para Sreebny la xerostomía se asocia frecuentemente a una disminución del flujo salival (hiposalivación). ${ }^{7}$

Así Bashkar señala que la caries dental es la enfermedad más común del ser humano. ${ }^{11}$ Domínguez, describe a la caries dental, como una secuencia de procesos de destrucción localizada en los tejidos duros dentarios que evoluciona en forma progresiva e irreversible. ${ }^{12}$ Por otro lado Newbrun explica los estados iniciales de la caries radicular con la desmineralización que ocurre en el cemento y en la dentina seguida por el rompimiento de fibras colágenas dentinales. Este rompimiento del colágeno está mediado por enzimas bacterianas y/o por la colagenasa mamalian producida por el huésped. Estas proteínas neutrales son secretadas como zymógenos inactivos y son activados comúnmente por enzimas bacterianas sobre las superficies radiculares expuestas, así las lesiones cariosas a menudo se expanden primero a lo ancho y no muestran límite definido. ${ }^{13}$ Las superficies radiculares se han encontrado más vulnerables al ataque ácido que el esmalte (Hoppenbrouwr). El valor crítico del $\mathrm{pH}$ para las superficies radiculares se estima que puede ser tan alto como 6.7 vs 5.4 para las superficies de esmalte. ${ }^{14}$

El objetivo de esta investigación es determinar la correlación entre flujo de salivación y caries corono-radicular en adultos mayores, en cuales se determinó las propiedades físico-químicas de la saliva ( el volumen y $\mathrm{pH}$ ) y la frecuencia de caries corono-radicular ya que estudios sobre la correlación entre el flujo salival total y la prevalencia de caries dental no han sido concluyentes.

\section{Material y Método}

El estudio fue de tipo analítico, observacional y transversal.

La investigación se realizó en una población de adultos mayores.

Se seleccionó una muestra de una población accesible de personas por conglomerados de los distritos de Lima: En Comas, fue el centro de salud ESSA-
LUD Merino Molina. En el distrito del Rímac en el asilo Cesar Canevaro, en el Distrito de La Victoria ESSALUD Nicolás de Piérola y en el Distrito de Carabayllo el Club de Adulto Mayor. El tamańo de la muestra seleccionada fue de 45 personas. se excluyeron a las personas con enfermedades sistémicas, infecciosas que tengan cierta gravedad y que tengan menos de diez dientes. También se seleccionó una muestra de 45 personas entre 16 -26 años de edad, estudiantes universitarios, grupo control para el estudio del volumen salival,

Se realizó una observación previa, para determinar la presencia de dientes en boca, debería tener un mínimo de 10 piezas. En la toma de saliva estimulada se aplicó la técnica de masticación de parafina por 5 minutos, la persona fue escupiendo paulatinamente la saliva en un depósito acondicionado. Inmediatamente se realizó la medición del $\mathrm{pH}$ y el volumen salival, para lo cual se empleó un pHchimetro electrónico y micropipetas. Se evaluó la higiene bucal, utilizando el Índice de Higiene Oral de Greene Vermellion (IHO) coloreando los dientes con violeta de genciana. Clínicamente se identificó la caries dental empleando espejos bucales y exploradores, las observaciones se anotaron en un odontograma acondicionado para anotar este tipo de caries, para ello me- dimos el COD y COS ( caries coronoradicular por diente y por superficie). Las personas fueron ubicadas en sillas para la observación de la caries, se utilizó una lámpara frontal para iluminar la boca e instrumentos manuales, (explorador y espejo bucal).

\section{Resultados}

Las personas comprendidas en nuestro estudio se encontraban entre $56 \mathrm{y}$ 86 años de edad con un promedio de 70,26 años y una $\pm 8,36$ donde el $96 \%$ estaban comprendidas 60 y 86 años de edad. La muestra estaba conformada por personas de ambos sexos, pero el sexo femenino tenia mayor representatividad $(71 \%$.)

El flujo salival en los adultos mayores se encontró con un volumen medio de 0,65 $\mathrm{ml} / \mathrm{min}( \pm 0,47)$ el volumen minimo en $5 \mathrm{~min}$ fue $0,5 \mathrm{ml}$ y máximo $11,7 \mathrm{ml}$. (ver Tabla N.o 1).

El pH salival en estas personas tuvo una media de 7,1 y $\pm 0,37$, lo cual determina un $\mathrm{pH}$ básico alcalino (ver tabla no 2 )

En nuestro estudio, los adultos mayores presentaron una media de 16,2 dientes presente en boca con $\pm 6,59$. El $95 \%$ tuvieron caries con una media de $5,8 \mathrm{y}$ \pm 3,34 (Tabla N.o 3)

Tabla N. ${ }^{\circ}$ 1. Flujo salival estimulada durante 5 minutos en adultos mayores, Lima 2011

\begin{tabular}{lccc}
\hline Flujo salival & Frecuencia & Porcentaje & $\begin{array}{c}\text { Porcentaje } \\
\text { acumulado }\end{array}$ \\
\hline $0,5-2 \mathrm{ml}$ & 16 & 35,6 & 35,6 \\
$2,1-4 \mathrm{ml}$ & 19 & 42,2 & 77,8 \\
$4,1-6 \mathrm{ml}$ & 6 & 13,3 & 91,1 \\
$6,1-8 \mathrm{ml}$ & 1 & 2,2 & 93,3 \\
$8,1-11,7 \mathrm{ml}$ & 3 & 6,7 & 100,0 \\
Total & 45 & 100,0 & \\
\hline
\end{tabular}

Tabla N. ${ }^{\circ}$ 2. $\mathrm{pH}$ salival de los adultos mayores en Lima 2011

\begin{tabular}{lccc}
\hline $\mathrm{pH}$ & Frecuencia & Porcentaje & $\begin{array}{c}\text { Porcentaje } \\
\text { acumulado }\end{array}$ \\
\hline $6,3-6,8$ & 9 & 20,0 & 20,0 \\
$6,81-7,0$ & 7 & 15,6 & 35,6 \\
$7,01-7,30$ & 16 & 35,6 & 71,1 \\
$7,31-7,95$ & 13 & 28,9 & 100,0 \\
Total & 45 & 100,0 & \\
\hline
\end{tabular}

Tabla N. ${ }^{\circ}$ 3. Caries dental, coro- radicular en adultos mayores, Lima 2011

\begin{tabular}{lccc}
\hline Caries & Frecuencia & Porcentaje & $\begin{array}{c}\text { Porcentaje } \\
\text { acumulado }\end{array}$ \\
\hline $0-2$ & 7 & 15,6 & 15,6 \\
$3-5$ & 15 & 33,3 & 48,9 \\
$6-9$ & 18 & 40,0 & 88,9 \\
$10-14$ & 5 & 11,1 & 100.0 \\
Total & 45 & 100,0 & \\
\hline
\end{tabular}


Tabla N. ${ }^{\circ}$ 4. Dientes cariados y obturados (CAO) en los adultos mayores, Lima 2011

\begin{tabular}{lccc}
\hline CAO & Frecuencia & Porcentajes & $\begin{array}{c}\text { Porcentajes } \\
\text { Acumulado }\end{array}$ \\
\hline $0-2$ & 6 & 13,3 & 13,3 \\
$3-5$ & 16 & 35,6 & 48,9 \\
$6-9$ & 14 & 31,1 & 80,0 \\
$19-17$ & 9 & 20.0 & 100,0 \\
Total & 45 & 100,0 & \\
\hline
\end{tabular}

De los dientes presente en boca los dientes afectados por caries (cariados y obturados) es el $95 \%$ con una media 6,37 y \pm 4.03 ( Tabla N.o4)

Del total de las superficies de los dientes presente, no se encontró caries solo en dos personas, la media de caries fue el 7,95 \pm 5,40 (Tabla N.o 5) $60 \%$ de las personas con una media de 2,48 y $\pm 1,64$; mientras que la caries en palatino se encontró en el 35,6\% de las personas, con una media de $1,5 \pm 0,81$. En la arcada inferior, se encontró en la superficie bucal caries en un $62 \%$ de las personas con una media de 2,67 y una $\pm 1,72$. El $62,2 \%$, en superficie lingual con una media de 2,67 y una $\pm 1,8$. Mientras que, en las caras proximales fueron afectadas el 57,7 \% de las personas y con una media de 4,53 $\pm 3,07$.

La higiene bucal de los adultos mayores fue mala, el valor mínimo encontrado fue de 1.7. (índice de Greene y Vermillon, malo: 1,5 - 3)

En relación al volumen salival de las persona adultos mayores y el de los jóvenes se encontró una diferencia al-
En la arcada superior, se observó caries vestibular (zona corono-radicular) en el

tamente significativa donde el Valor $\mathrm{T}$ es 3,88 con $\mathrm{p}=0.00$ (ver Tabla N. ${ }^{\circ}$ 6).

\section{Discusión}

El flujo salival que tienen las personas se va modificando conforme va aumentando la edad, haciéndose notorio en las personas adultas mayores, eso lo que pudimos identificar en el presente estudio, la media del flujo salival estimulado en personas jóvenes fue de $1,05 \mathrm{ml} / \mathrm{m}$. con una Ds 0.47 . Mientras que en los adultos mayores con un promedio de edad de 70,26 años fue de $0.65 \mathrm{ml} / \mathrm{m} \mathrm{y} \pm 0,48$ donde el valor $T$ dio un $\mathrm{p}<0.000$ nos determinó diferencia estadísticamente significativa.

Medina M, Merino L. y Gorodner L. ${ }^{3}$ afirman que una producción constante de saliva, con un promedio de flujo es de $1-3 \mathrm{ml} / \mathrm{min}$ es secretada con características especificas en respuesta a un grupo diversos de estímulos. Esto es muy parecida a la encontrada en los jóvenes universitarios, pero muy por encima de la encontrada en personas adultas mayores de Lima.

El pH de la saliva estimulada en personas mayores fue de un promedio de

Tabla N. ${ }^{\circ}$ 5: Superficies dentarias atacadas por caries dental en adultos mayores, Lima 2011

\begin{tabular}{lcrc}
\hline $\begin{array}{l}\text { Caries por } \\
\text { Superficies }\end{array}$ & Frecuencias & Porcentaje & $\begin{array}{r}\text { Porcentaje } \\
\text { Acumulada }\end{array}$ \\
\hline $0-5$ & 17 & 37,8 & 37,8 \\
$6-10$ & 14 & 31,1 & 68,9 \\
$11-14$ & 10 & 22,2 & 91,1 \\
$15-24$ & 4 & 8,9 & 100,0 \\
Total & 45 & 100,0 & \\
\hline
\end{tabular}

Tabla N. 6 Grupos etarios y volumen salival estimulado en cinco minutos

\begin{tabular}{llccc}
\hline & Grupos etarios & $\mathrm{N}$ & Media & Desviación \\
$\begin{array}{l}\text { Error estándar d } \\
\text { la media }\end{array}$ & & & Estándar \\
\hline $\begin{array}{l}\text { Volumen } \\
0,35865\end{array}$ & Adultos mayores & 45 & 3,2729 & 2,40592 \\
$\begin{array}{l}\text { Salival } \\
0.35957\end{array}$ & Jóvenes & 45 & 5,2433 & 2,41208 \\
\hline
\end{tabular}

7.10 y una \pm 0.38 , podemos ver que tienen una saliva básica alcalina encontrándose dentro de los parámetros de la normalidad según lo especificado por la literatura ${ }^{17}$. Otro resultado relevante es que el $100 \%$ de las personas de tercera edad tienen mala higiene bucal $(1,8$ 3) según el índice de higiene simplificado Green y Vermillon.

Al tratar de seleccionar la muestra encontramos un alto porcentaje de personas de tercera edad desdentadas totalmente otras con remanentes radiculares y escasos dientes, que no fueron seleccionados para el estudio, dentro de los criterios de exclusión era que las personas tengan un mínimo de 10 piezas dentarias en boca, la obtención de la muestra apta para el estudio fue muy difícil , en las muestra las personas tuvieron un promedio de 16 pzas. Nuestros resultados obtenidos coinciden con las proposiciones de Krasse B. (1989), el sostiene que cuando el rango de secreción salival está reducido, las lesiones cariosas a menudo aparecen en la unión cemento-esmalte. En general, tales lesiones cariosas pueden ser distinguidas fácilmente por la erosión o reabsorción idiopática y como norma tienen una base dura. ${ }^{11}$

Podemos ver que la presencia de caries se encuentra muy alta, y que los dientes presente en boca fueron afectados en su totalidad (95\%).

Pero no se pudo determinar si el volumen salival deficiente es el riesgo para desarrollar caries por la presencia de placa bacteriana muy abundante y la retracción gingival. Según las teorías, la presencia de estos elementos, son de alto riesgo para que los adultos mayores desarrollen caries. Podemos hacer mención a McNamara TF(1998) quien dice que la incidencia de caries radicular aumenta a medida que la población envejece, y que las exposiciones radiculares por recesión gingival o pérdida del nivel de inserción, han aumentado con el envejecimiento. ${ }^{12}$ El estudio concuerda con respecto a la correlación del bajo flujo salival y la elevada frecuencia de caries dental, concordando también con Banderas, J; González, M. et al (15) que mencionan que sujetos con "boca seca" frecuentemente presentan un alta prevalencia de caries dental en contraste con aquellos con flujo salival alto. Sin embargo, los estudios sobre la correlación entre el flujo salival total y la prevalencia de caries dental no han sido concluyentes $^{15}$ Según los resultados obtenidos podemos consideran a las personas adultas mayores como perso- 
nas de alto riesgo para desarrollar caries dental por presentar muchos elementos de riesgo.

\section{Conclusiones}

- Las personas adultas mayores presentan muy mala higiene bucal.

- El flujo salival en los adultos mayores es bajo a muy bajo, con un $\mathrm{pH}$ salival básico- alcalino

- La caries corono-radicular es muy elevada con presencia mayor en las superficie proximal de los dientes de los adultos mayores.

- Podemos considerar a los adultos mayores de alto riesgo para desarrollar caries dental en la superficie corono-radicular.

Se recomienda realizar un estudio de cohortes sobre el flujo salival en personas adultas mayores con mala y buena higiene bucal. También entre los problemas para estudiar en esta población son la retención de prótesis total en adultos mayores y la relación entre Higiene bucal y el desarrollo de neumonías en adultos mayores.

Agradecimiento: Al Vicerrectorado de Investigación de la Universidad Nacional Mayor de San Marcos por el apoyo económico que nos brindó para la ejecución del estudio.

\section{Referencias bibliográficas}

1. Martínez AG, Ledesma MC, Sandoval MR, Sanzon Ortega MC. Factores que propician el establecimiento de los procesos cariosos. Pract Odontol 1993;14(5):31-5.

2. Katz, Simon; McDonald, James et al. Odontología preventiva en acción. $3^{\mathrm{a}}$ ed. México DC: Panamericana; 1991. pp. 171-194.

3. Loesche WJ, Schork A, Terpenning Ms, Chen YM, Stoll J. Factors which influence levels of selected organisms in saliva of older individuals. J Clin Microbiol 1995;33(10):2550-7.

4. Masamura K, Inaba R, Iwata H. Salivary calcium and total protein in relation to dental caries. Nippon Eiseigaku Zasshi 1995;50(4):886-92.

5. Martínez Díaz M, Reyes Macías JF, Noyola Frías MA. Usos diagnósticos de la saliva. ADM 1992;49(3):15560.

6. Barrancos Mooney J. Operatoria Dental. $4^{\mathrm{a}}$ ed. México DC: Panamericana; 2006. 1345p.

7. Sreebny LM. Saliva in health and disease: an appraisal and update. Int Dent J. 2000; 50:140-61

8. Van der Reijden WA, Veerman ECI, Nieuw-Amerongen AV. Shear ratedependent viscoelastic behaviour of human glandular saliva. Biorheology 1993;30:141-52.

9. Krasse B. Caries risk: A practical guide for assessment and control. Chicago:Quintessence;1985. p1132.

10. Ramamurthy NS., Schroeder KI., McNamara TF. et al. Root-surface caries in rats and humans: Inhibition by a non-antimicrobial property of tetracyclines. Adv Dent Res.1998;12:43-50.

11. Bhaskar SN. Patología Bucal. 6a ed. Buenos Aires: El Ateneo;1984. p123-8.

12. Domínguez FV en Cabrini RL. Anatomía patológica bucal. Buenos Aires: Mundi; 1980. 235 p.

13. Newbrun E. Cariology. 3rd ed. Chicago: Quintessence books; 1989. p. 67-70.

14. Aamdal-Scheie A., Luan WM. Plaque $\mathrm{pH}$ and Microflora of Dental Plaque on sound and carious root surfaces. J Dent Res. 1996;75(11):1901-1908.

15. Banderas, JA; González M. et al. Flujo y concentración de proteínas en saliva total humana. Salud Pública Méx. 1997;39:433-41

16. Medina M, Merino L y Gorodner L. Utilidad de la saliva como fluido diagnostico, Universidad Nacional del Nordeste, Argentina 2002. 